QUADERNI Quaderni

Communication, technologies, pouvoir

82 | Automne 2013

L'hôpital à l'épreuve de la performance économique

\title{
Elvina Fesnau, \\ Le poste à transistors à la conquête de la France, la radio nomade (1954-1970)
}

Jean-Jacques Cheval

\section{OpenEdition}

\section{Journals}

Édition électronique

URL : https://journals.openedition.org/quaderni/749

DOI : 10.4000/quaderni.749

ISSN : 2105-2956

Éditeur

Les éditions de la Maison des sciences de l'Homme

Édition imprimée

Date de publication : 5 octobre 2013

Pagination : 99-104

Référence électronique

Jean-Jacques Cheval, «Elvina Fesnau,

Le poste à transistors à la conquête de la France, la radio nomade (1954-1970) », Quaderni [En ligne], 82

Automne 2013, mis en ligne le 05 octobre 2015, consulté le 08 mars 2022. URL : http://

journals.openedition.org/quaderni/749; DOI : https://doi.org/10.4000/quaderni.749 


\section{Compte rendu}

\section{Le poste à transistors à la conquête de la France, la radio nomade (1954-1970) \\ Elvina Fesnau}

INA Éditions, coll. "Médias histoire", Paris, 2011

par Jean-Jacques Cheval

Université de Bordeaux 3 -

Michel de Montaigne

GRER, Groupe de Recherches et

d'Etudes sur la Radio,

L'ouvrage d'Elvina Fesneau traite d'un épisode essentiel, mais méconnu de l'histoire des médias en France, la naissance du poste de radio à transistors dans notre pays au milieu des années 50 , puis son déploiement dans les années suivantes, jusqu'à la fin des années 60 , établissant son devenir hégémonique en la matière.

Durant ces quinze années, les Français s'approprient une innovation venue des États-Unis et mise pour la première fois sur le marché, en 1954, outreAtlantique, avec le poste Regency commercialisé par l'entreprise Texas Instruments. En France, après un démarrage industriel et commercial difficile, le poste à transistors se convertit en un objet déjà présent dans plus de $20 \%$ de foyers en 1962, puis dans plus de 70\% en 1969, plaçant la France au tout premier rang des pays européens. Constatant ce passage relativement rapide du poste à transistors au statut d'objet de consommation courante ou de masse, Elvina Fesneau, à bon droit, peut écrire que «Le poste à transistors devient, d'une certaine façon, l'un des symboles de ces "Trente Glorieuses" » (p. 307).

L'ouvrage, ainsi que l'annonce d'emblée l'auteure, n'a pas vocation à étudier la technologie du composant électronique « transistor », mais l'histoire du poste de radio à transistors dans le cadre français, à travers des dimensions industrielles et économiques qui prévalent dans le texte, mais sans oublier les facteurs politiques, sociologiques et culturels qui accompagnent son appropriation. La précision est nécessaire car, comme on le sait, la sémantique 
commune va rapidement mêler l'appellation des pièces électroniques en question avec les récepteurs qu'elles équipent. Dans le langage courant, le transistor est devenu synonyme d'un poste de radio mobile.

L'étude est issue d'une thèse d'histoire contemporaine, soutenue en 2009 à l'Université de Paris 1 - Panthéon Sorbonne, réalisée sous la direction de l'historien Jacques Marseille (1945-2010). Elle en a la rigueur, la précision et la solidité et s'est nourrie d'une abondante documentation.

Les sources sont en partie constituées par les archives d'André Danzin, l'un des dirigeants de la Compagnie Générale de Télégraphie sans Fil (CSF), ancien vice-président directeur général, qui supervisa en son temps la production des transistors et le lancement du premier récepteur français. Elles ont été complétées par des données et ressources collectées auprès de l'INSEE, dans les archives de l'Inathèque ou celles de Radio France et au Centre des archives contemporaines de Fontainebleau. Elles sont aussi tirées des dossiers du ministère des Finances, d'archives bancaires, de celles de la Fédération nationale de l'industrie électronique conservées à Chambre de Commerce et d'Industrie de Paris ; du dépouillement de revues professionnelles ou d'émissions de radio et de télévision où il était question $\mathrm{du}$ poste à transistors. Issus de ces recherches, de nombreux tableaux, graphiques et séries statistiques viennent appuyer le récit et la démonstration. Enfin l'auteure a conduit de nombreux entretiens avec des collectionneurs, hommes de radio, industriels, ou encore distributeurs de transistors.

Le livre suit une démarche historique chronologique partant des années 50 et allant jusqu'à la fin des années 60 . Cette trame linéaire classique est croisée de nombreuses problématiques auxquelles se confronte l'auteure pour expliquer comment le poste à transistors a su susciter l'engouement des consommateurs : Quels furent les rôles de l'offre et de la demande en la matière? Quels paramètres techniques, industriels et économiques ont facilité, ou au contraire, retardé temporairement le succès de ce nouvel appareil ? Quelle fut l'importance ou l'impact de l'action des politiques, des pouvoirs publics face à ce phénomène ?

L'ouvrage se compose de trois parties. La première analyse la construction et le lancement du premier poste à transistors français par la Compagnie Générale de Télégraphie Sans Fil, le Solistor mis en vente en 1956. Il 
constituait la première proposition française dans ce domaine et fut un échec au demeurant. S'il fut pionnier, il n'atteint pas les foules, et Elvina Fesneau décortique les causes de ce premier faux départ au prisme des archaïsmes et résistances, des difficultés technologiques inhérentes aux prototypes. Ce revers fut lié aussi à des erreurs stratégiques d'alliance de la CSF (avec Philips en l'occurrence) ou encore la conséquence des pratiques de tests surprenantes des premiers appareils ; ces essais furent confiés aux seuls cadres supérieurs et dirigeants de l'entreprise, qui n'étaient guère représentatifs du marché potentiel envisagé. «Les bonnes idées ne suffisent pas toujours à contribuer à la réussite du lancement d'un nouveau produit sur le marché», juge Elvina Fesneau et « en matière d'innovation, l'on dit souvent qu'il vaut mieux être suiveur que précurseur» (p. 106). De plus, si l'initiative industrielle existait, l'État français, focalisé sur la télévision, ne s'était pas réellement intéressé aux récepteurs à transistors.

Dans une deuxième partie, le décollage du nouveau produit est détaillé pendant la période nommée des " Quatre Glorieuses », soit les années allant de 1958 à 1962. La conquête du marché s'amorce avec encore des inégalités, mais déjà les programmateurs prennent conscience du tournant qui s'annonce et la production des programmes radiophoniques eux-mêmes se modifie. Dans cette partie, Elvina Fesneau s'engage aussi dans des essais d'études sémiologiques de publicités (dont de nombreuses sont reproduites) visant à promouvoir l'objet et qui forment la base d'un nouvel imaginaire de la radio dans lequel les notions de mobilité, d'autonomie occupent une place de choix.

Dans le chapitre 6, Elvina Fesneau s'interroge sur l'existence d'usages spécifiques du poste à transistors. Elle évoque ses rapports à l'automobile. Dans un premier temps, le récepteur portatif à transistors s'emporte dans les voitures et remplace les rares et chers autoradios à lampes. Dans le même temps la $R T F$ et Europe $n^{\circ} 1$, entre autres, créent plusieurs émissions destinées aux auditeurs en voiture. Ainsi, en 1958, Europe $n^{\circ} 1$ lance Les voix de la nuit, une émission animée par des jeunes femmes, qui apportent une présence amicale aux automobilistes, puis c'est le lancement, par la radio publique, de Inter Service Route en 1959, avec les premiers essais de radio guidage.

Si des interrogations persistent encore sur la fiabilité, les qualités sonores des postes à transistors, l'élargissement de la gamme des appareils disponibles vont balayer ces doutes y compris pour un modèle qui conquiert rapidement 
le public : le poste à transistors de poche, attractif par sa maniabilité et son prix. En 1960, un poste d'appartement à transistors, représente plus de 3 jours de travail d'un ouvrier français, mais moins de deux jours pour un récepteur de poche. "La miniaturisation des appareils et la baisse de leur prix désacralisent "le poste de famille" qui trônait jusqu 'ici sur le buffet de la salle à manger car "c'est maintenant une petite boîte que l'on met dans sa poche”» (p. 207), relève l'auteure.

Concernant cette période, Elvina Fesneau va à l'encontre d'une idée courante d'une corrélation entre le lancement avec succès de la station Europe $n^{\circ} 1$ et l'apparition des nouveaux appareils récepteurs. De même, elle minimise les nouveaux usages de la radio qui seraient liés intrinsèquement à la nouvelle technologie, si ce n'est pour noter une influence sur la consommation journalière ou saisonnière du média. On se surprend à lire qu'à cette époque, en 1961, l'INSEE évaluait la durée d'écoute journalière des Français à 1 heure et 42 minutes, alors qu'elle est de près de 3 heures en semaine, cinquante plus tard.

En parallèle, on s'amuse des rappels des préventions que suscitent les postes à transistors dans leurs usages publics, leur présence est jugée parfois abusive. Un journaliste de l'époque commentait : «La transistomanie est une véritable maladie. » (p. 192) Les autorités préfectorales s'emploient à réguler cette vogue bruyante en limitant l'usage des récepteurs au nom de la tranquillité et du droit au silence, notamment dans les taxis, dans les trains ou sur les plages.

La troisième partie du livre couvre la banalisation du poste à transistors avec les corrélations sociales, économiques et culturelles qui l'accompagnent et les nouvelles interrogations qui en découlent, notamment de la part des politiques. Le marché s'envole, alors que la croissance de la demande est amplifiée par la hausse du niveau de vie qu'accompagne l'inflation et de nouvelles baisses des prix des récepteurs.

Les jeunes, les adolescents, deviennent véritablement les cibles des constructeurs et distributeurs de poste radio à transistors, objet identificateur, social et individualisé à la fois, symbole de nouvelle culture et de nouvelle forme de loisir. Souvenons-nous qu'en 1966, Nino Ferrer en avait fait le refrain d'une chanson, Les cornichons : "On est parti, samedi, dans une grosse voiture, Faire tous ensemble un grand pique-nique dans la nature, En emportant des paniers, des bouteilles, des paquets, Et la radio ! » ; un 
tube que l'on écoutait à la radio justement. Aux États-Unis la radio avait accompagné assez étroitement la dispersion d'une expression musicale jusqu'alors ghettoïsée dans l'ensemble de la société : le rock'n'roll. En France, les sons du Swinging London et de la musique yéyé se trouvent un vecteur non exclusif mais essentiel à travers les nouveaux récepteurs radio.

Si la France marque un net retard en matière d'utilisation de la modulation de fréquence c'est essentiellement par une pénurie de programmes diffusés selon cette norme. Il ne s'agit pas d'une limitation technologique mais bien du contingentement de la radiodiffusion dans le cadre du monopole d'État en vigueur.

Dans son dernier chapitre, Elvina Fesneau s'arrête sur les rapports ambivalents de la radio et de la politique, particulièrement à l'occasion des événements de Mai 1968. Ici, on n'est peut-être pas obligé de la suivre totalement quand elle pose que « le poste à transistors permet d'échapper à l'emprise du pouvoir politique » (p. 303). Ce constat est sans doute nourri d'une vision partielle de l'usage de la radio durant cette période. Car si, par exemple, l'auteure rappelle justement que l'utilisation de postes à transistors « a servi aux manifestants pour connaître les déplacements des forces de police » (p. 303). L'inverse était tout aussi vrai ; le préfet de Police de Paris, Maurice Grimaud, en a témoigné. De même, quand Elvina Fesneau écrit : " cette crise politique fut emblématique du rôle particulier des postes à transistors car ils échappent au contrôle et provoquent des réactions de masse » (p. 302), c'est oublier que, le 30 mai 1968, ce fut par une allocution, uniquement radiodiffusée, et une manifestation de masse, mais nullement spontanée, très orchestrée, que le général de Gaulle et ses partisans reprirent le contrôle du cours des choses. Si mai 68 semble annoncer des modifications quant à la volonté de « prendre la parole », il faudra encore attendre une dizaine d'années pour que la France connaisse l'explosion des radios libres. Le potentiel libératoire de l'outil technique était réel sans doute, mais il ne s'est pas révélé unilatéralement et immédiatement. Elvina Fesneau le rappelle d'ailleurs elle-même dans sa conclusion, en citant Dominique Wolton : " il ne faut pas confondre performance technique et nouvelles mœurs sociétales » (p. 308).

En conclusion et au-delà des ces appréciations qui sont l'objet de débats, il faut souligner tout l'intérêt du travail d'Elvina Fesneau. À la croisée de plusieurs disciplines, l'Histoire et plus précisément l'Histoire économique 
et contemporaine, les Sciences de l'information et de la communication, il participe à un évident regain d'intérêt pour le sujet radiophonique au sein des recherches en Sciences humaines et sociales. Il était devenu monnaie courante de déplorer le déficit d'études sur ce média, ce n'est plus le cas, ni en France - notamment grâce au Groupe de Recherches et d'Etudes sur la Radio (GRER - http://radiography.hypotheses.org) dont Elvina Fesneau est l'une des fondatrices - ni à l'étranger si l'on veut bien se départir d'un tropisme national. Les publications récentes de nombreux livres collectifs en témoignent et pour n'en évoquer que quelques unes, citons : Radio Content in the Digital Age: The Evolution of a Sound Medium, édité par Angeliki Gazi, Guy Starkey, et Stanislaw Jedrzejewski (Bristol (UK) / Chicago (USA), Intellect, 2011); Histoire de la radio : Ouvrez grand vos oreilles ! édité sous la direction d'Hervé Glevarec (Paris : Musée des arts et métiers / Silvana Editoriale, 2012), le catalogue d'une exposition qui s'est tenue au CNAM, à Paris, durant un semestre en 2012 ; l'Ebook : Radio Evolution : Conference Proceedings, édité par Madalena Oliveira, Pedro Portela et Luís António Santos de l'Université de Braga au Portugal (http://www.lasics.uminho.pt/ ojs/index.php/radioevolution); The Palgrave Handbook of Global Radio, de John Allen Hendricks Ed. (New-York /Basingstoke : Palgrave Macmillan, 2012), souhaitant établir un panorama actuel de la radio au niveau mondial ; Radio - Community, Challenges and Aesthetics, édité par Grażyna Stachyra (Maria Curie-Skłodowska University Press, Lublin, Pologne, 2013)...

Ces nouvelles recherches mettent souvent l'accent sur les transformations à l'œuvre de la radio et des technologies à l'heure du numérique. Elvina Fesneau conclut justement son livre avec quelques pages pertinentes consacrées à la numérisation de la radio. En cela, elle est fidèle à un conseil maintes fois répété, repris ici de l'historien états-unien Robert Darnton et placé en exorde de l'ouvrage " Toute tentative pour sonder l'avenir tout en affrontant les problèmes du présent devrait se fonder, je le crois, sur l'étude du passé. » (p. 8) En effet, on peut judicieusement penser que l'exemple du bouleversement technique qu'a constitué l'arrivée puis le succès du poste à transistors, le renouvellement rapide du parc des récepteurs, les transformations des usages qui s'en sont suivies, peuvent aujourd'hui nous éclairer sur les changements qui s'annoncent et qui pourront constituer, ou non, une permanence ou une nouvelle renaissance de la radio, un média décidément " résilient 》 ainsi qu'il est désigné lors d'un colloque international de recherche qui s'est tenu à Londres en septembre 2013 (http://radioresearch2013.sunderland.ac.uk). 